\title{
Die Vorteile der Fibulaköpfchenexstirpation bei Unterschenkelamputation nach persönlichen Erfahrungen.
}

\author{
Von \\ Dr. A. Lehrnbecher, \\ sssistenzarzt der chir. Abteilung des Stidt. Krankenhauses Nürnberg.
}

Hit 2 Ahbildungen in Text.

(Eingegangen am 12. Mai 1922.)

Trotzdem die Fragen über Amputationen und Prothesen-Anpassung nicbt mehr so alituell sind wie während und kurz nach dem Kriege, möchte ich doch nicht unterlassen, über meine persönlichen Erfahrungen mit einer Operation zu berichten, die anscheinend keine allzugroße Verbreitung gefunden hat die aber gerade bei Friedensamputationen in manchen Fälen vorzügliche Resultate verspricht; es ist dies die Exstirpation des Fibulaköpfohens bei Cnterschenkelamputation.

Diese Operation wurde im Jahre 1915 von Prof. $v$. Batey empfohlen, im Jahre 1917 berichtete derselhe bereits über 25 mit gutem Erfolg operierte Fille. Auch von anderen (hirurgen und Orthopäden wurde die Operation vorgenommen (Erlacher), in der Orthop. Klinik König Ludwig-Haus Würzburg wurde sie aurh ron dem Narhfolger Prof. r. Baerer's. Prof. Port, in ciner ganzen Reihe von Fällen vorgenommen.

Teehnik nach v. Bacyer:

,Blutlecre, hinter dem Wadenbeinköpfchen $10 \mathrm{~cm}$ langer Schuitt. der mit den Nachoperationswunden nicht in Verbindung stehen darf, Durchtrennung der Weichteile im Bereiche der lateralen Fläche der Fibula. Den Peronaeus schont man stets bei langen Stümpfen, um nicht die ron ihm imervierte Mus. kulatur zu schädigen, die trotz der Inaktivitütsatrophie immer noch in gewissem Grade die Knochen polstert. Bei kurzen Stümpfen reseziert man besser den Nerven, nachdem man ihn weit hervorgezogen hat. Die Fibula wird schräg von außen unten nach oben medial durchmeilselt und nun ausgeschält, die Bizepssehne bleibt in Verbindung mit den Weichteilen des Unterschenkels. Naht der Muskulatur. Faszie und Haut."

Es ist vielleicht am zweckmäßigsten, die Vorteile der Operation an Hand meiner eigenen Krankengeschichte zu erläutern: 
In Juni 1913 wurle mir als Bataillonsarzt bei einer Teiloffenive durch Granatsplitter der rechte Fuß zerwhettert. Da der Anuriff ziemlich weit über das feindliche Grabennetz vorgetragen und der Rücktransirort dementsprechend Jangwierig war, bounte die $A b$ setzung erst aun nächsten Tage in einem Fekllazarett vorgenommen werden. Nichtsdestuweniger unternahm der (hirurg des Lazarettes (Stabsarzt d. P. Dr. Jansen) in hervorragender Weise das Wagnis, bei der Exartikulation an den Jalleolen die noch verwendbare Fersenhant abzulöen und unter misgicbiger Drainage durch einige Situationsnähte zu fixieren.

Durch die unvermeidbare Infektion schnitten späterhin die Fäden durch, der Lappen retrahierte sich teilwcise. Bei siner nach 2!: Monaten im König Ludwig-Haus Würzburg von Prof. v. Baeyer vorgenommenen zweiten Operation konnte aber unter Wegnahme von Knochen wieder zur vollständigen Deckung der Stumpffläche gebracht werden, so

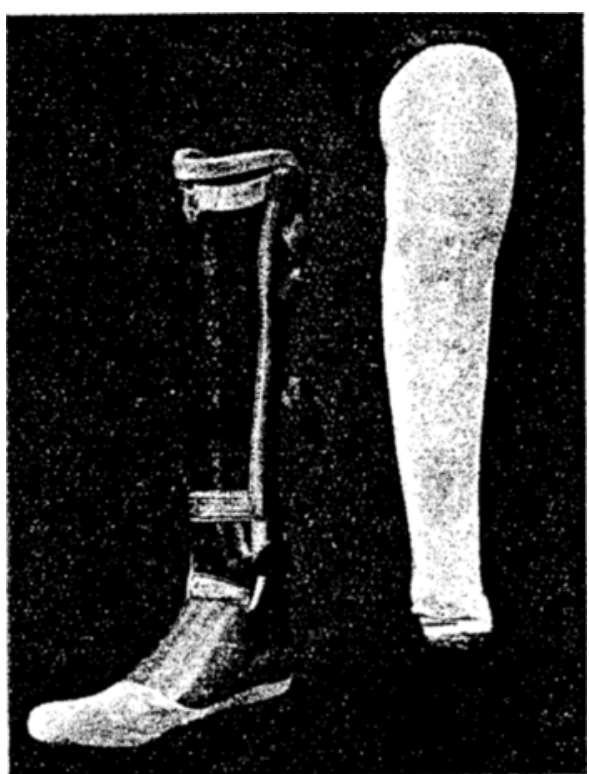

Abb. 1 . daß jetzt Verhältnise vorliegen, ähnlich wie bei der Exartikulation nach Sy me; nur verläuft die Absetzungslinie nicht melir in Hiohe rles oberen sprunggelenkes. sondern zwei Qnerfinger oberhalb der Knöchelspitzen.

Nach Abklingen einer mäir stark aufgetretenen Wundeiterung (latente Infektion) konnte ich nach fünf Wochen zum erstenmal mit dem $r$ Bacyerwehen Sitzstock ausgphen, nach weiteren drei Wochen bekam ich das Bohelfsbein.

Bei der rweiten Operation wurcle zu Beginu der sitzung in Techiadilusleitungsanärthesia die Exstirpation des Fibulaköpfchens vorgenommen, ich hatte in den seitclem rerflossenen $31 / 2$.Tahren Gelegenheit die rotziugliche Wirkung der Uperation zu erfahren.

Jetzirerer Befund:

Absetzung zwei (puerfinger oberhalb der Knörhelspitzen, die Verkürzung beträgt 11 cm.

Die Stumpffläche ist ron der vorzüglich ernährten Fersenhaut gerleckt, an der Innenseite eine tiefeingezogene Narbe (Drainage), an der dubenseite eine längere flächenförmige Narbe. (J)ecubitus durch Suhienenoliuck.)

Im oberen Drittel iles Tnterschenkels an der AuBenseite fine $10 \mathrm{~cm}$ lange nach hinten konvexe Operationwwunde. An Stelle der Prominenz cles Fibulakopf chens ist hier cine Eindellung vorhanden (vgl. Photographie).

Waden-tmiang links: $38 \mathrm{im}$

, $"$ rechts: $31, \tilde{j},$.

['mfang in Höbe des Fibulaköpfchens links: $35 \mathrm{~cm}$.

Trnfing an der entsprechenden Stelle rechts: $28,5 \mathrm{~cm}$.

Ich trauce ein sehr einfach konstruiertes Kunstbein mit verstellbaren sohnallen. wie es sonst bei Pirogoff-Amputierten gebräuchlich ist mit fest fixiertem Knöhelgelenk und BlockfilzrorderfuB (nach Marks) ${ }^{1}$ ).

Die von Prof. $\because$. Baeyer bei der Fibulaköpfehen-Exstirpation frstrebten Vorteile bestchen ror allem darin, daß man durch seine Eutfernung die Ursache vicler Beschwerden beseitigt und eine neue gut ausnützhare Stutzflache schafft.

1) Dieser FuB läßt einen mindestens ebenso gnten Gang zu wie die Konstruktionen mit Gelenken, es fallen dabei die leidigen Reparaturen durch Achsenbruch fort. 
Wem ein Prothesengïnger sich mit den Eigenschaften seines Apparates rertraut gemacht und die Kinderkrankheiten des Kunstbeingehens überwunden hat, ist es in der Folgezeit meist nur noch das Aufschenern der Haut, das ihn grhunfähig macht. Eine ganz besonders ausgeprägte Prärdilektionsstelle ist das Fibulaköptchen, namentlich wenn es bei starker Entwicklung und bei der unvermeidbaren Nuskelatrophie stärker nach außjen prominiert. Die Haut wird zwischen Fibula- und Lederhïlse der Prothese cingepreBt und scheuert sich lcicht durch, besonders im Sommer. In manchen Fällen bilden sich auch an diescr Stelle Schleimbeutel, die sich entzünden können und inzidiert wrorden müssen. Unter Unständen karm auch hier ein sehr unangenehmer Druck auf den Peronaeus vorhanden sein.

In den beiclen ersten Jahren habe ich mich an sehr vielen Stcllen des Cnterschenkels aufgescheuert, an der Vorderscite der Tibia, in der Nähe cles Stumpfes. im oberen Drittel an der Innenseite, hier mußte cimmal ein Schleimbentel inzidiert werclen. Niemals aber habe ich an der sonst so gefürchteten Stelle an der Aufenscite die geringsten Unannehmlichkeiten gehabt.

In gleicher Weise sind die Torteile der Schaffung einer neuen Stützfläche leieht einzusehen.

Bei einer Cntersohenkelamputation werden, un den Ausführungen Gocht's zu folgen, zur Belastung herangezogen

1. die untere Stumpfflïche,

2. clie ganze Zirkumferenz rler seitlichen Stumpfflïche,

3. der obere Linterschenkelteil

a) die mediale Auslarlung des Schienbeinknorren,

b) die Tuberositas Tibiae,

c) der untere Teil der Kniescheibe.

dazu kommt noch eine mehr oder mincler breite fülse am Oberschenkel, bei maneben Fälen mit Tubersitz, viele Lnterschenkel-Amputierte haben auch cine Aufhängevorrichtung an der Schulter notwendig.

Wie Borchardt sich ausdriickt, treten die einzelnen Stützflichen vikaricrend für einander ein; je mehr vorhanden sind, desto weniger wird die cinzelne beansprucht und unterliegt keinem so dauernden Druck.

Auf dem Röntgenbild ist gut zu sehen, wio sich infolge Fohlens des Fibulaköpfchens der laterale Kondylus der Tibia weit vorwölbt und eine ne ue Haftflïche für dic Prothese biklet. Der neugebildete obere Teil der Fibula ist weit nach innen gebogen. Besonders anschaulich lïßt ein Vergleich mit einem normalen Röntgenbilid die Veründerung erkenmen.

Dieses erstrebenswerte Ziel wird dadurch erreicht, daß I2 'Tage nach der Operation um das obere Ende des Cnterschenkelstumpfes ein etwa $5 \mathrm{~cm}$ breites Band geschlungen wird, das im Bereich der Narbe ein rundes Polster besitzt. Dadurch wird vermieden, daß die Knochenneubildung eine unerwünschte Form annimmt. Das Anziehen des Bandes darf nicht brüsk vorgenommen werden, sondern muß unter allmählicher Steigerung erfolgen, da der Druek auf den Peronaeus nicht angenehm ist.

Natürlich tritt die volle Ausnützung der neuen Stützfläche nicht sofort. ein, man lernt überhaupt erst allmählich die verschieden hohe Wertigkeit der 
einzelnen Belastungsstellen abschätzen unsl ausnützen, auch ist die Haut an diesen Stellen erst nach längerer Zeit so widerstandsfähig, um den .immerhin erheblichen Druck auszuhalten. Ich habe die verschiedenen dhstufungen des Unterschenkelkumstheines kennen gelernt, erst Behelfsbein mit Schulteraufhängung und Tubersitz, dann Lnterschenkelbein mit Oberschenkelhülse, seit zwei Jahren trage ich das oben beschriebene kurze Cnterschenkelbein.

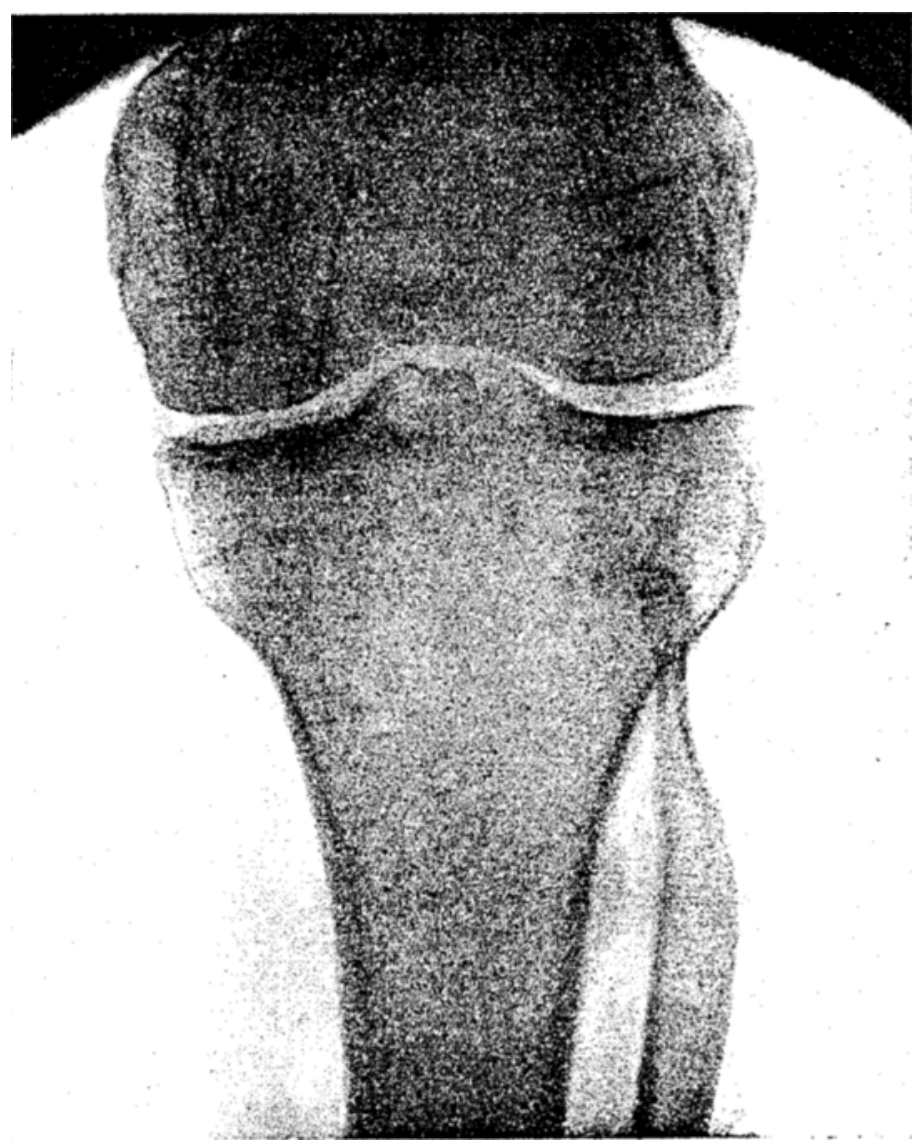

Abb. 2 .

Daß nir das Tragen einer Unterschenkelhülse ohne Oberschenkelteil möglich ist, verdanke ich außer der nengeschaffenen Stützlläche einer dritten Folge der Operation.

Dureh den Ausfall des oberen Fibulaendes entsteht an der Außenseite im proximalen Drittel des Cnterschenkels eine Lücke, in die das obere Ende der Prothesenhülle hineinpaß3t, weiter distal ist wieder Fibula vorhanden und die stärker entwickelte Wadenmuskulatur. Hierdurch wird ein Abgleiten der Prothese unmöglich gemacht, was bei Vorhandensein des Wadenbeinköpfchens und darlureh bedingtem erikeblich großeren Unfang des Unterschenkels im 
oberen Drittel bei Treppensteigen und Springen unvermeidlich wäre; der Gang wäre durch das beständige Schlottern des Kunstbeines unsicher und man könnte sich durch abruptes Hinfallen verletzen. Bei Pirogoff- und ChopartStümpfen gewährt das kolbige distale Ende hinreichenden Schutz gegen das tbrutschen des Kunstbeines, bei tiefen Unterschenkelamputationen ist es sonst nötig, den Oberschenkel durch eine Hülse mitzufassen.

Ich möchte ausdrüchlich darauf hinweisen, daß die kurze Prothese doch cinen recht erheblichen Vorteil darstellt. Es fällt die durch den Hülsendruck bedingte Atrophie der Oberschenkelmuskulatur aus, das Knie hat freien Spielraum, der Apparat ist nicht so schwer und kostspielig.

Dazu kommt noch ein weiterer Umstand, den ich gerade in praktischer Beziehung sehr hoch einschätzen möchte. Die Amputierten haben sich in der Regel leicht damit abgefunden, daß sie zu Höchstleistungen. wie sie Personen mit gesunden Gliedern ausführen, nicht mehr befähigt sind. Es sind vor allem die kleinen fortwährenden Lnanmehmlichkeiten, die sie an ihre Ausnahmestcllung exinnern, das lästige An- und Ausziehen der Prothese, die Abnützung der Kleider u. dgl. Dies fällt aber bei einem Unterschenkelbein vollständig weg, man schlüpft hinein wie in einen großen Stiefel, die oben erwähnten Unbequemlichkeiten sind nicht vorhanden.

Selbstverständlich ist hier ein Individualisieren geboten. Für Landwirte und Schwerarbeiter ist es unter Unständen zweckmäßiger ein Bein mit Oberschenkelhülse zu tragen, um alle zur Verfügung stehenden Stützflächen auszunützen, Stadtbewohner werden eher mit einer kleinen Prothese auskommen. Bei größeren Ausflügen kann man ein Reserve-Bein mit sich führen um durch ein Umwechseln neue Druckverhältnisse zu schaffen, da natürlich niemals zwei Kunstbeine in völlig gleicher Weise passen.

Unbedingt notwendig ist allerdings, der durch das Tragen der Unterschenkelhülse bedingten Atrophie durch eine fortwährende psychogene Stumpfgymnastik entgegen zu wirken, wie sie Gocht in seinem Buch über künstliche Glieder beschrieben hat.

Noch auf einen weiteren Vorteil der Methode sei hingewiesen, den v. Ba ayer erwähnt hat, der bei mir jedoch woniger in Betracht kam, in anderen Fällen aber von grober Wichtigkeit ist. Durch die Entfernung des Köpfchens rückt die Fibula etwas in die Höhe, in vielen Fällen ron Nachamputation, wo die Haut zur Deckung der Stumpfflache knapp ist, wird dieselbe dann ausreichen und man braucht nichts von der Tibia zu opferm.

Hs soll nicht unterlassen sein, darauf hinzuweisen, daß gewisse Kontraindikationen bestehen. Vor allem sei ausdrücklich betont, da $B$ die Vornahme der Operation eine gewisse Technik erfordert, da die Schonung des Nervus peronaeus nicht ganz leicht ist. Ferner ist darauf zu achten, daß die Operation nur bei reinen Wundverhältnissen rorgenommen wird, da sonst Vereiterung des Kniegelenkes eintreten kann, wie sie Hofstätter erlebt hat. Die Gefahr des Aufrührens einer latenten Infektion ist vor allem bei hohen Unterschenkelamputationen vorhanden, wo die Stumpffläche in der Nähe des Fibulaköpfchens liegt. Hier ist aber nach den Ausführungen v. Baeyers die Operation nicht so notwendig, da die kurzen Stümpfe meist nur zur Bewegung der Prothese herangezogen werden können und weniger für das Tragen derselben in Betracht 
116 A. Lehrn becher: Die Vorteile d. Fibulaköpfchenexstirpation b. Unterschenkelamputat.

kommen; v. Baeyer hat dieser Indikation immer Rechnung getragen und nie Mißerfolge erlebt.

Bei Kriegsunterschenkelamputationen wird sich auch bei Fibulaköpfchen-Fxstirpation das Wegbleiben der Oberschenkelhülse nur ermöglichen lassen, wenn es sich um eine ganz tiefe Absetzung handelt, und die Stumpffläche in höherem Grade belastungsfähig ist. Unter allen Umständen aber läßt sich ein Freimachen von der lästigen und unschönen Schulteraufhängung und dem Tubersitz erreichen.

Ein in hohem Grade segensreiche Wirkung aber wird die FibulaköpfchenExstirpation bei Friedensamputationen haben, bei denen die Heilungsberlingungen der Wunde und die Aussichten für Tragfähigkeit ungleich günstiger sind wie bei den primär infizierten Kriegsverletzungen.

Ganz besonders in Betracht kommt hier die Anwendung bei Amputationen, die einen tragfähigen Stumpf erzielen, bei der osteoplastischen Stumpfversorgung nach Bitr. der aperiostalen Methode nach Bunge, der tendinoplastischen Stumpfiversorgung nach Wilms, der freien Faszienplastik auf die Stumpfflache nach Ritter, zumal wenn nach diesen Operationen das Stumpftraining nach Hirsch vorgenommen wird.

Vielleicht ließe sich auch mit großem Vorteile eine Kombination mit der Oehleckerschen Wethode der Bildung kolbiger Lnterschenkclstumpte schaffen oder mit der von Orth und Fränkel empfohlenen Bolzung der Stümpfe dureh Knochentransplantate. Es wäre die Möglichkeit gegeben, in einer Sitzung das Fibulaköpfehen zu entfernen und es dann dicht oberhalb des Stumpfes einzubolzen, die Nachteile der nicht gleichmäßigen Gestalt des entnommenen Knochens ließe sich durch Spaltung desselben und Einbolzung von verschiedenen Seiten her vermeiden. Fränkel hat ja clie Spaltung besonders empfohlen, da , der Einfluß des Knochenmarkes frei gemacht wird ".

Es wäre so denkbar, ein schlechthin ideales Resultat zu errielen, unten cine tragfïhige kolbig verdickte Auftrittsfläche, oben eine Verschmälerung zum Festhalten der Prothese und gute Stütz- und Haftflächen für die Hülse.

\section{Zusammenfassung.}

Die Exstirpation des Fibulaköpfchens bei Untersehenkelamputation ist in vielen Fällen von außerordentlichem Vorteil: es wird die Prädilektionsstelle für Druckläsion ausgeschaltet, eine neue Stützfläche geschaffen, bei supramalleolären Absetzungen das Abrutschen der Prothese verhindert und manch. mal eine Nachamputation durch das Höherrücken der Fibula wesentlich erleichtert. In Verbindung mit den modernen plastischen Methoden der Stumpfversorgung wird sich ein ideales Resultat erreichen lassen.

\section{Literatur.}

1. v. Baeyer, Münch. med. Wochenschr. 1915. Nr. 44. - 2. Derselbe, Münch. ined. Wochenschr. 1917. Nr. 24. - 3. Borchardt, Die Stumpfversorgung an der unteren Extremität, Ersatzglieder und Arbeitshilfen. Berlin 1919. - 4. Bier, Braun, Kü m mel, Chirurg. Operationslehre. 3. Aufl. - 5. Fränkel, Dtsch. Zeitschr. f. Chir. 166. - 6. Gocht, Radike und Schede, Kinstliche Glieder. 2. Aufl. - 7. Hofstätter, Wien. klin. Wochenschrift 1916. Nr. 35. - 8. Oehlecker, Zentralbl. f. Chinurg. 1918. Nr. 15. 